Article

\title{
On the Extrinsic Principal Directions and Curvatures of Lagrangian Submanifolds
}

\author{
Marilena Moruz ${ }^{1,+}+\left(\mathbb{D}\right.$ and Leopold Verstraelen ${ }^{2, *,+}$ \\ 1 Department of Mathematics, KU Leuven, Celestijnenlaan 200B-Box 2400, 3001 Leuven, Belgium; \\ marilena.moruz@kuleuven.be \\ 2 PiT and CiT, Department of Mathematics, KU Leuven, Celestijnenlaan 200B-Box 2400, 3001 Leuven, Belgium \\ * Correspondence: leopold.verstraelen@kuleuven.be \\ + These authors contributed equally to this work.
}

Received: 31 July 2020; Accepted: 4 September 2020; Published: 8 September 2020

\begin{abstract}
From the basic geometry of submanifolds will be recalled what are the extrinsic principal tangential directions, (first studied by Camille Jordan in the 18seventies), and what are the principal first normal directions, (first studied by Kostadin Trenčevski in the 19nineties), and what are their corresponding Casorati curvatures. For reasons of simplicity of exposition only, hereafter this will merely be done explicitly in the case of arbitrary submanifolds in Euclidean spaces. Then, for the special case of Lagrangian submanifolds in complex Euclidean spaces, the natural relationships between these distinguished tangential and normal directions and their corresponding curvatures will be established.
\end{abstract}

Keywords: extrinsic principal tangential directions; principal first normal directions; Lagrangian submanifolds

\section{The Extrinsic Tangential Principal Directions of Submanifolds}

For general submanifolds $M^{n}$ of dimension $n(\geq 2)$ and of co-dimension $m(\geq 1)$ in Euclidean spaces $\mathbb{E}^{n+m}$, Jordan [1] studied the extrinsic curvatures $c_{u}^{T}(p)$ at arbitrary points $p \in M$ in arbitrary tangential directions determined by vectors $u \in T_{p} M,\|u\|=1$. These are the curvatures $c_{u}^{T}(p)=$ $\left(d \varphi_{u} / d s\right)^{2}(0)$, whereby $\varphi_{u}(s) \in[0, \Pi / 2]$ denotes the angle in $\mathbb{E}^{n+m}$ between the tangent spaces $T_{p} M$ at $p$ and $T_{q} M$ at a nearby point $q \in M$ in the direction $u$ of $M$ at $p, s$ being an arclength parameter of a curve $\gamma$ on $M$ from $p=\gamma(0)$ in the direction $u=\gamma^{\prime}(0)$ to $q=\gamma(s)$. He defined the tangential principal curvatures $c_{1}^{T}(p) \geq c_{2}^{T}(p) \geq \ldots \geq c_{n}^{T}(p) \geq 0$ of a submanifold $M^{n}$ in $\mathbb{E}^{n+m}$ at $p$ as the critical values of the tangential Casorati curvature function at $p$, that is of the function $c^{T}(p): S_{p}^{n-1}(1)=\left\{u \in T_{p} M \mid\|u\|=1\right\} \rightarrow \mathbb{R}^{+}$: $u \mapsto c_{u}^{T}(p)$, and, he defined the tangential principal directions of a submanifold $M^{n}$ in $\mathbb{E}^{n+m}$ at $p$ as the directions in which these critical values of the curvatures $c_{u}^{T}(p)$ are attained and proved these directions to be mutually orthogonal, say to be determined by orthonormal vectors $f_{1}, f_{2}, \ldots, f_{n} \in T_{p} M$.

In the first step of his original fundamental studies of the geometry of submanifolds, Trenčevski [2-5] re-considered this work of Jordan, and, later, Stefan Haesen and Daniel Kowalczyk and one of the authors [6] basically re-did this. In the latter paper were followed the 1890 Casorati's views on the intuitively most natural scalar valued curvatures "as such" of surfaces $M^{2}$ in $\mathbb{E}^{3}$ (and in [6,7], some tangential and normal kinds of curvature of Riemannian submanifolds were named after Casorati). Accordingly, in [6], the above tangential Casorati curvatures rather came up as $c_{u}^{T}(p)=\left(d \psi_{u} / d s\right)^{2}(0)$, whereby $\psi_{u}(s)$ denotes the angle in $\mathbb{E}^{n+m}$ between the normal spaces $T_{p}^{\perp} M$ at $p$ and $T_{q}^{\perp} M$ at a nearby point $q$ in the direction of $u$ (as was already known by Jordan, $\left.\psi_{u}=\varphi_{u}\right)$. As shown by Trenčevski, the extrinsic principal unit tangential vector fields $F_{1}, F_{2}, \ldots, F_{n}$ of a submanifold $M^{n}$ in $\mathbb{E}^{n+m}$, and their corresponding tangential Casorati principal curvature functions $c_{1}^{T}, c_{2}^{T}, \ldots, c_{n}^{T}: M \rightarrow \mathbb{R}^{+}: p \mapsto c_{1}^{T}(p), c_{2}^{T}(p), \ldots, c_{n}^{T}(p)$ are essentially the orthonormal eigen vector fields, 
and the corresponding eigen functions of the symmetric linear Casorati operator $A^{C}=\sum_{\alpha} A_{\alpha}^{2}$, whereby $A_{\alpha}=A_{\xi_{\alpha}}$ are the shape operators of $M^{n}$ in $\mathbb{E}^{n+m}$ for arbitrary orthonormal normal frame fields $\xi_{1}, \xi_{2}, \ldots, \xi_{m}$ on $M^{n}$ in $\mathbb{E}^{n+m}$, such that $A^{C} F_{i}=c_{i}^{T} F_{i}, i \in\{1,2, \ldots, n\}, \alpha \in\{1,2 \ldots, m\}$; (the intrinsic principal tangential directions and their corresponding curvatures of a submanifold $M^{n}$ in $\mathbb{E}^{n+m}$, of course, being its Ricci principal directions and curvatures).

From the above, in particular, one may notice that for hypersurfaces $M^{n}$ in $\mathbb{E}^{n+1}$, the extrinsic principal tangential directions are "the classical" principal directions of these hypersurfaces, whereas $\left\{c_{1}^{T}, c_{2}^{T}, \ldots, c_{n}^{T}\right\}=$ $\left\{k_{1}^{2}, k_{2}^{2}, \ldots, k_{n}^{2}\right\}, k_{1}, k_{2}, \ldots, k_{n}$, being the classical principal curvatures of these hypersurfaces, correspond to Kronecker's extension of Euler's theory of the curvature of surfaces $M^{2}$ in $\mathbb{E}^{3}$ to hypersurfaces $M^{n}$ in $\mathbb{E}^{n+1}$ for all dimensions $n \geq 2$.

\section{Felice Casorati's Study of Surfaces $M^{2}$ in $\mathbb{E}^{3}$}

Casorati [8] defined his extrinsic scalar-valued curvature $C(p)$ of a surface $M^{2}$ in $\mathbb{E}^{3}$ at one of its points $p$ as follows. On $M^{2}$, consider a small geodesic circle $\gamma_{\Delta_{\rho}}$ centered at $p$ with radius $\Delta_{\rho}$. Let $q$ be any point on $\gamma_{\Delta_{\rho}}$, and consider the geodesic $\delta$ from $p$ to $q$ parametrised by arclength, such that $p=\delta(0)$ and $q=\delta\left(\Delta_{\rho}\right)$; at $p$, this geodesic points in the tangential direction $\delta^{\prime}(0)=u$ to $M^{2}$ at $p$. Let $\eta(p)$ and $\eta(q)$ be the unit normal vectors on the surfaces $M^{2}$ in $\mathbb{E}^{3}$ at $p$ and at $q$, respectively, corresponding to a choice of unit normal vector field $\eta$ around $p$ on $M^{2}$ in $\mathbb{E}^{3}$. Then, in Casorati's words, and according to our common sense, the angle $\Delta \psi_{u}$ between $\eta(p)$ and $\eta(q)$ measures well how much the surface $M^{2}$ at $p$ curves in the direction $u$; the more the surface curves in the direction $u$, the larger this angle. Then, joining all the points $\delta\left(\Delta \psi_{u}\right)$ that thus correspond to all the points $q$ on the geodesic circle $\gamma_{\Delta_{\rho}}$ around $p$, associated with $\gamma_{\Delta_{\rho}}$, one obtains on $M^{2}$ a closed curve $\Gamma_{\Delta_{\rho}}$ (which actually passes through $p$ whenever, at $p$, the surface is not curved at all in some tangential directions $u$ ). Hence, according to our common sense, the bigger or the smaller the area's $A\left(\Gamma_{\Delta_{\rho}}\right)$ enclosed on $M^{2}$ by the curves $\Gamma_{\Delta_{\rho}}$ as compared to the area's $A\left(\gamma_{\Delta_{\rho}}\right)$ of the geodesic discs on $M^{2}$ bounded by the geodesics $\gamma_{\Delta_{\rho}}$, the more or the less the surface $M^{2}$ "as such" in $\mathbb{E}^{3}$ is curved at $p$. It was along this line of thought that Casorati defined his curvature of a surface $M$ in $\mathbb{E}^{3}$ at $p$ as $C(p)=\lim _{\Delta_{\rho} \rightarrow 0}\left(A\left(\Gamma_{\Delta_{\rho}}\right) / A\left(\gamma_{\Delta_{\rho}}\right)\right)$, and he proved that $C(p)=\frac{1}{2} \operatorname{tr} A^{2}(p)=\frac{1}{2}\left(k_{1}^{2}+k_{2}^{2}\right)(p)$ $=\frac{1}{2}\|h\|^{2}(p)$, whereby $k_{1}$ and $k_{2}$ are Euler's principal curvatures, $A$ is the shape operator of $M^{2}$ corresponding to $\eta$ and $h$ is the second fundamental form of $M^{2}$ in $\mathbb{E}^{3}$.

At this stage, it might be not amiss to add the following comment. In the definition of his curvature $C$, Casorati followed the common basic, i.e., from the original geometrical definitions of the curvature $K$ of Gauss and the mean curvature $H$ of Germain via ratios of well-chosen areas related to the surfaces $M^{2}$ in $\mathbb{E}^{3}$. For $K(p)$, these ratios concern regions on $M^{2}$ around $p$ and their corresponding spherical images, and, for $H(p)$, these ratios are for discs centered at $p$ in $T_{p} M$ and for the portions of the corresponding circular cylinders perpendicular to $T_{p} M$ in between $T_{p} M$ and the surface $M^{2}$ in $\mathbb{E}^{3}$ itself. While for the curvatures of Germain and Gauss, this lead to the first two elementary symmetric functions of $k_{1}$ and $k_{2}, H=\frac{1}{2} \operatorname{tr} A=\frac{1}{2}\left(k_{1}+k_{2}\right)$ and $K=\operatorname{det} A=k_{1} k_{2}$, Casorati's geometrical definition of his curvature yielding that $C=\frac{1}{2} \operatorname{tr} A^{2}=\frac{1}{2}\left(k_{1}^{2}+k_{2}^{2}\right)$ lead to the third elementary symmetric function of Euler's principal curvatures.

\section{The First Normal Principal Directions of Submanifolds}

Trenčevski determined the maximal possible dimensions of the osculating spaces of all orders for submanifolds $M^{n}$ in $\mathbb{E}^{n+m}$ and, moreover, in the related succesive normal spaces, of all orders, and determined appropriate orthonormal frames of principal normal vector fields and corresponding principal normal curvatures. For our present purpose, it may suffice here to restrict within this grand theory to what is stated in Theorem 1 of [7]: "The first principal normal directions of a submanifold $M^{n}$ in $\mathbb{E}^{n+m}$ are the normal directions of $M^{n}$ in $\mathbb{E}^{n+m}$ in which the normal Casorati curvatures of $M^{n}$ attain their $m_{1}$ (=dimension of the first normal space $N_{1}$ ) non-zero critical values." The first normal space $N_{1}$ of $M^{n}$ in $\mathbb{E}^{n+m}$ is the subspace of the total normal space $T^{\perp} M$ of $M^{n}$ in $\mathbb{E}^{n+m}$ given by $N_{1}=\operatorname{Im} h=\{h(X, Y) \mid X, Y \in T M\}$, whereby $h$ is the 
second fundamental form of the submanifold $M$, or, $N_{1}$ is the orthogonal complement in $T^{\perp} M$ of the subspace consisting of all normals $\xi$ with vanishing shape operators $A_{\xi}$, or, equivalently, with vanishing normal Casorati curvature $c_{\xi}^{\perp} ; N_{1}=\left\{\xi \in T^{\perp} M \mid A_{\xi}=0\right\}^{\perp}=\left\{\xi \in T^{\perp} M \mid c_{\xi}^{\perp}=0\right\}^{\perp}$, such that the first osculating space of $M^{n}$ in $\mathbb{E}^{n+m}$ is given by $T M \oplus N_{1}$.

The considerations of Casorati on surfaces $M^{2}$ in $\mathbb{E}^{3}$ that were recalled above can straightforwardly be taken over to general submanifolds $M^{n}$ in $\mathbb{E}^{n+m}$ (and to general submanifolds $M^{n}$ in ambient general Riemannian spaces $\tilde{M}^{n+m}$, for that matter) cfr. [6]. In [6], a.o. one may find that the Casorati curvature (as such) of a submanifold $M^{n}$ in $\mathbb{E}^{n+m}$ equals the arithmetic mean of its tangential principal Casorati curvatures: $C=\frac{1}{n}\|h\|^{2}=\frac{1}{n} \operatorname{tr} A^{C}=\frac{1}{n} \sum_{\alpha} \operatorname{tr} A_{\alpha}^{2}=\frac{1}{n} \sum_{i} c_{i}^{T}$. Moreover, it seems not without interest to observe that $C_{\xi}(p)=\frac{1}{n} \operatorname{tr} A_{\xi}^{2}(p)$ is the Casorati curvature (as such) at $p$ of the projection $M_{\xi p}^{n}$ of the submanifold $M^{n}$ in $\mathbb{E}^{n+m}$ onto the $(n+1) D$ subspace $\mathbb{E}^{n+1}$ of $\mathbb{E}^{n+m}$, which is spanned by $T_{p} M=$ $\mathbb{R}^{n}$ together with the normal line $[\xi(p)], \xi$ being any unit normal vector field on $M^{n}$ in $\mathbb{E}^{n+m}$, and, hence, that $C_{\xi}(p)=\frac{1}{n} \sum_{i} c_{\xi_{i}}^{T}(p)$, i.e., $C_{\xi}(p)$ is the arithmetic mean of the tangential Casorati curvatures $c_{\tilde{\xi} i}^{T}$ of this projected hypersurface $M_{\xi}^{n}$ at $p$ (for some general considerations relating the contemplation and the theory of submanifolds, see [9]). The functions $c_{\xi}^{\perp}: \mathbb{S}^{m-1}(1)=\left\{\xi \in T^{\perp} M \mid\|\xi\|=1\right\} \rightarrow \mathbb{R}^{+}$: $\xi \mapsto c_{\tilde{\xi}}^{\perp}=\frac{1}{n} \operatorname{tr} A_{\xi}^{2}$ are called the normal Casorati curvatures of $M^{n}$ in $\mathbb{E}^{n+m}$; more precisely, the normal Casorati curvature of $M^{n}$ in $\mathbb{E}^{n+m}$ in the direction determined by a unit normal vector field $\xi$ is defined as $c_{\xi}^{\perp}=\frac{1}{n} \operatorname{tr} A_{\xi}^{2}$.

In the total, $m D$ normal space $T^{\perp} M$ of $M^{n}$ in $\mathbb{E}^{n+m}$, consider the following symmetric linear operator $a: T^{\perp} M \rightarrow T^{\perp} M: \xi \mapsto a(\zeta)=\frac{1}{n}\|\zeta\| \sum_{\alpha}\left(\operatorname{tr} A_{\zeta} A_{\alpha}\right) \xi_{\alpha}$; (in [10], Bang-Yen Chen basically introduced this operator in the study of the submanifolds for which $a(\vec{H})=\overrightarrow{0}, \vec{H}$ being the mean curvature vector field of $M^{n}$ in $\mathbb{E}^{n+m}$, submanifolds which later were called Chen submanifolds; in this respect, see also [11,12]). And, by the principal axes theorem, there exists an orthonormal frame $\eta_{1}, \eta_{2}, \ldots, \eta_{m_{1}}, \eta_{m_{1}+1}, \ldots, \eta_{m}$ of eigen vector fields for this operator $a: T^{\perp} M \rightarrow T^{\perp} M\left(m_{1}=\operatorname{dim} N_{1}\right)$, with corresponding eigen functions $c_{1}^{\perp}=\frac{1}{n} \operatorname{tr} A_{\eta_{1}}^{2} \geq c_{2}^{\perp}=\frac{1}{n} \operatorname{tr} A_{\eta_{2}}^{2} \geq \ldots \geq c_{m_{1}}^{\perp}=\frac{1}{n} \operatorname{tr} A_{\eta_{m_{1}}}^{2}>c_{m_{1}+1}^{\perp}=$ $\operatorname{tr} A_{\eta_{m_{1}+1}}^{2}=\ldots=c_{m}^{\perp}=\operatorname{tr} A_{\eta_{m}}^{2}=0$. The normal vector fields $\eta_{1}, \eta_{2}, \ldots, \eta_{m_{1}}$ span the first normal space $N_{1}$ of $M^{n}$ in $\mathbb{E}^{n+m}$ and, following Trenčevski, are called the first principal normal vector fields of the submanifold $M^{n}$ in $\mathbb{E}^{n+m}$ with corresponding first-principal normal curvatures $c_{1}^{\perp} \geq c_{2}^{\perp} \geq \ldots \geq c_{m_{1}}^{\perp}>0$. So, with indices $\alpha_{1} \in\left\{1,2, \ldots, m_{1}\right\},\left\{\eta_{\alpha_{1}}\right\}$ is an orthonormal frame field of the first normal space $N_{1}$ for which $a\left(\eta_{\alpha_{1}}\right)=c_{\alpha_{1}}^{\perp} \eta_{\alpha_{1}}$, whereby $c_{\alpha_{1}}^{\perp}=\frac{1}{n} \operatorname{tr} A_{\alpha_{1}}^{2}(>0)$ are the principal normal Casorati curvatures of $M^{n}$ in $\mathbb{E}^{n+m}$.

\section{The Principal Tangent and the First Principal Normal Directions of Lagrangian Submanifolds}

From Section 16: Totally real and Lagrangian submanifolds of Kähler manifolds of Chen's contribution on Riemannian submanifolds in [11], is taken the following: "The study of totally real submanifolds of a Kähler manifold from differential geometric points of views was initiated in the early 1970's (by Bang-Yen Chen and Koichi Ogiue [13]—the authors). A totally real submanifold $M$ of a Kähler manifold $\tilde{M}$ is a submanifold such that the almost complex structure $J$ of the ambient manifold $\tilde{M}$ carries each tangent space of $M$ into the corresponding normal space of $M$, that is, $J\left(T_{p} M\right) \subset T_{p}^{\perp} M$ for any point $p \in M$. (...) A totally real submanifold $M$ of a Kähler manifold $\tilde{M}$ is called Lagrangian if $\operatorname{dim}_{\mathbb{R}} M=\operatorname{dim}_{\mathbb{C}} \tilde{M}$. 1-dimensional submanifolds, that is, real curves in a Kähler manifold are always totally real. For this reason, we only consider totally real submanifolds of dimension $\geq 2$. (...) For a Lagrangian submanifold $M$ of a Kähler manifold $(\tilde{M}, g, J)$ the tangent bundle TM and the normal bundle $T^{\perp} M$ are isomorphic via the almost complex structure J of the ambient manifold. In particular, this implies that the Lagrangian submanifold has flat normal connection if and only if the submanifold is a flat Riemannian manifold".

To continue in our aim to aim for simplicity and concreteness of presentation, (although, clearly, the following matters do hold more generally), we next restrict our attention to the real $n$ dimensional totally real submanifolds $M^{n}$ of the complex $n$ dimensional complex Euclidean spaces $\tilde{M}^{n}=\mathbb{C}^{n}=\left(\mathbb{E}^{2 n}, \tilde{J}\right)$, that is, to the Lagrangian submanifolds $M^{n}$ in $\mathbb{C}^{n}$, thus having $\tilde{J}(T M)=T^{\perp} M$ and $\tilde{J}\left(T^{\perp} M\right)=T M$, 
$\tilde{J}$ being the complex structure of the Kaehler manifold $\tilde{M}^{n}$. On $M$ in $\tilde{M}$, tangential vector fields will be denoted by $X, Y, Z, \ldots$ and normal vector fields by $\xi, \eta, \zeta, \ldots$. Further, let $\tilde{g}$ and $\tilde{\nabla}$, respectively, $g$ and $\nabla$, be the metrics and the corresponding Riemannian connections on $\tilde{M}$ and $M$, respectively. The equations of Gauss and of Weingarten are given by

$$
\begin{aligned}
& \tilde{\nabla}_{X} Y=\nabla_{X} Y+h(X, Y), \\
& \tilde{\nabla}_{X} \xi=-A_{\mathcal{\zeta}}(X)+\nabla_{X} \frac{1}{\xi},
\end{aligned}
$$

whereby $\nabla^{\perp}$ is the normal connection and $h$ the second fundamental form and $A_{\xi}$ the shape operator with respect to $\xi$ of the submanifold $M$ in $\tilde{M}$, so that

$$
\tilde{g}(h(X, Y), \xi)=g\left(A_{\tilde{\zeta}}(X), Y\right) .
$$

Applying the complex structure $\tilde{J}$ to (1), it follows that

$$
\tilde{J}\left(\tilde{\nabla}_{X} Y\right)=\tilde{J}\left(\nabla_{X} Y\right)+\tilde{J}(h(X, Y)),
$$

while writing (2) out for $\xi=\tilde{J} Y$, it follows that

$$
\tilde{\nabla}_{X}(\tilde{J} Y)=-A_{\tilde{J} Y}(X)+\nabla_{X}^{\perp}(\tilde{J} Y) .
$$

By the parallelity of $\tilde{J}, \tilde{\nabla} \tilde{J}=0$, or, still, $\tilde{J}\left(\tilde{\nabla}_{X} Y\right)=\tilde{\nabla}_{X}(\tilde{J} Y)$, the left-hand sides in (4) and (5) are equal, and, hence, in particular, the tangential components of the right-hand sides in (4) and (5) are also equal:

$$
\tilde{J}(h(X, Y))=-A_{\tilde{J} Y}(X) .
$$

Writing out (3) for $\xi=\tilde{J} Z$, it follows that

$$
\tilde{g}(h(X, Y), \tilde{J} Z)=g\left(A_{\tilde{J} Z}(X), Y\right),
$$

which, by (6), leads to

$$
\tilde{g}(h(X, Y), \tilde{J} Z)=g(-\tilde{J}(h(X, Z)), Y) .
$$

Since $\tilde{J}$ is almost complex, $\tilde{J}^{2}=-I$, and since $\tilde{g}$ is Hermitian, so that $\tilde{g}(\tilde{J} \tilde{V}, \tilde{J} \tilde{W})=\tilde{g}(\tilde{V}, \tilde{W})$ for all vector fields $\tilde{V}$ and $\tilde{W}$, and, hence, in particular, for $\tilde{V}=-\tilde{J}(h(X, Z))$ and $\tilde{W}=Y$, (8) becomes

$$
\begin{aligned}
\tilde{g}(h(X, Y), \tilde{J} Z) & =g\left(-\tilde{J}^{2}(h(X, Z)), \tilde{J} Y\right) \\
& =g(h(X, Z), \tilde{J} Y) .
\end{aligned}
$$

In view of its crucial importance in what comes next, we have worked out in detail this property from [13], as obtained in (9), which may be stated as follows. For all tangential vector fields $X, Y, Z$ on a Lagrangian submanifold $M^{n} \subset \mathbb{C}^{n}\left(\tilde{M}^{n}\right)$ :

$$
\tilde{g}(h(X, Y), \tilde{J} Z)=\tilde{g}(h(X, Z), \tilde{J} Y)=\tilde{g}(h(Y, Z), \tilde{J} X) .
$$

For any tangential orthonormal frame field $\mathcal{F}=\left\{E_{1}, E_{2}, \ldots, E_{n}\right\}$ on a Lagrangian submanifold $M^{n}, \tilde{\mathcal{F}}=$ $\left\{E_{1}, E_{2}, \ldots, E_{n}, \xi_{1}=\tilde{J} E_{1}, \xi_{2}=\tilde{J} E_{2}, \ldots, \xi_{n}=\tilde{J} E_{n}\right\}=\left\{E_{i}, \xi_{i}=\tilde{J} E_{i}\right\},(i, j, k, \alpha, \beta \in\{1,2, \ldots, n\})$ is a corresponding adapted orthonormal frame field of $\mathbb{C}^{n}\left(\tilde{M}^{n}\right)$ along $M^{n}$. The local coordinates of the operator $A^{C}: T M \rightarrow T M$ of Casorati and of the operator $a: T^{\perp} M \rightarrow T^{\perp} M$ of Trenčevski with respect to such frame fields $\tilde{\mathcal{F}}$ are given by 


$$
\begin{aligned}
A_{i k}^{C} & =\left(\sum_{\alpha} A_{\alpha}^{2}\right)_{i k}=\sum_{\alpha}\left(A_{\alpha}^{2}\right)_{i k} \\
& =\sum_{\alpha} \sum_{j} h_{i j}^{\alpha} h_{j k}^{\alpha}
\end{aligned}
$$

and

$$
\begin{aligned}
a_{\alpha \beta} & =\operatorname{tr}\left(A_{\alpha} A_{\beta}\right) \\
& =\sum_{i} \sum_{j} h_{i j}^{\alpha} h_{j i}^{\beta},
\end{aligned}
$$

whereby $h_{i j}^{\beta}$ are the local coordinates of the symmetric second fundamental form $h: T M \times T M \rightarrow T^{\perp} M$. Therefore, $E_{i}$ determines a Casorati principal tangential vector field on $M^{n}$ in $\mathbb{C}^{n}$ with corresponding principal tangential Casorati curvature $c_{i}^{T}$ if and only if

$$
\forall k \neq i: A_{i k}^{C}=\sum_{\alpha} \sum_{j} h_{i j}^{\alpha} h_{j k}^{\alpha}=0,
$$

whereby

$$
c_{i}^{T}=A_{i i}^{C}=\sum_{\alpha} \sum_{j}\left(h_{i j}^{\alpha}\right)^{2},
$$

and, $\xi_{\alpha}=\tilde{J} E_{\alpha}$ determines a first principal normal vector field, or, first Casorati principal normal vector field (as these vector fields later on also might be termed), on $M^{n}$ in $\mathbb{C}^{n}$ with corresponding principal normal Casorati curvature $c_{\alpha}^{T}$ if and only if

$$
\forall \beta \neq \alpha: a_{\alpha \beta}=\sum_{i} \sum_{j} h_{i j}^{\alpha} h_{j i}^{\beta}=0,
$$

whereby then

$$
c_{\alpha}^{\perp}=a_{\alpha \alpha}=\sum_{i} \sum_{j}\left(h_{i j}^{\alpha}\right)^{2} .
$$

Written in local coordinates, the above property (10) amounts to

$$
\forall i, j, k: h_{i j}^{k}=h_{i k}^{j}=h_{j k}^{i}
$$

so that, from (13) and (15), and, from (14) and (16), in particular, we may conclude the following.

Theorem 1. Let $M^{n}$ be a Lagrangian submanifold of the complex Euclidean space $\mathbb{C}^{n}$ (or of any Kaehler manifold $\left.\tilde{M}^{n}\right)$. Then, a tangential vector field $T$ is a tangential principal Casorati vector field with corresponding tangential Casorati principal curvature $c^{T}(>0)$ if and only if $N=\tilde{J} T$ is a normal principal Casorati vector field—whereby $\tilde{J}$ is the complex structure of $\mathbb{C}^{n}$ (or, of the ambient Kaehler space, $\tilde{M}^{n}$ )—with corresponding normal Casorati principal curvature $c^{\perp}=c^{T}(>0)$.

Theorem 2. Let $M^{n}$ be a Lagrangian submanifold of the complex Euclidean space $\mathbb{C}^{n}$ (or, of any ambient Kaehler manifold $\left.\tilde{M}^{n}\right)$ with first normal space of maximal dimension $\left(m_{1}=\operatorname{dim} N_{1}=n=c o-\operatorname{dim} M\right)$. Then, $M^{n}$ admits an adapted orthonormal frame field $\tilde{\mathcal{F}}=\left\{F_{1}, F_{2}, \ldots, F_{n}, \eta_{1}=\tilde{J} F_{1}, \eta_{2}=\tilde{J} F_{2}, \ldots, \eta_{n}=\tilde{J} F_{n}\right\}$ in $\mathbb{C}^{n}\left(\tilde{M}^{n}\right)$, of which the $n$ tangential vector fields are the principal Casorati tangential vector fields and of which the $n$ normal vector fields are the principal Casorati normal vector fields of $M^{n}$ in $\mathbb{C}^{n}\left(\tilde{M}^{n}\right)$, and the corresponding tangential and normal principal curvatures are equal, $\left(\forall i: c_{i}^{T}=c_{i}^{\perp}\right)$.

Author Contributions: Investigation, M.M. and L.V. The authors contributed equally to this work. All authors have read and agreed to the published version of the manuscript.

Funding: M.Moruz is a postdoctoral fellow of the Research Foundation-Flanders (FWO). 
Conflicts of Interest: The authors declare no conflict of interest.

\section{References}

1. Jordan, C. Généralization du théorème d'Euler sur la courbure des surfaces. C. R. Acad. Sci. Paris 1874, 79, 909-912.

2. Trenčevski, K. Principal directions for submanifolds imbedded in Euclidean spaces of arbitrary codimension. Proc. Third Intern. Workshop on Diff. Geom. Appl. First Ger. Rom. Semin. Geom. Gen. Math. 1997, 5, 385-392.

3. Trenčevski, K. New approach for submanifolds of the Euclidean space. Balkan J. Geom. Appl. 1997, 2, 117-127.

4. Trenčevski, K. Geometrical interpretation of the principal directions and principal curvatures of submanifolds. Diff. Geom. Dyn. Syst. 2000, 2, 50-58.

5. Trenčevski, K. On the osculating spaces of submanifolds in Euclidean spaces. Kragujevic J. Math. 2012, 36, $45-49$.

6. Haesen, S.; Kowalczyk, D.; Verstraelen, L. On the extrinsic principal directions of Riemannian submanifolds. Note Mat. 2009, 29, 41-51.

7. Verstraelen, L. Geometry of submanifolds I. The first Casorati curvature indicatrices. Kragujevic J. Math. 2013, 37, 5-23.

8. Casorati, F. Mesure de la courbure des surfaces suivant l'idée commune. Acta Math. 1890, 14, 95-110. [CrossRef]

9. Verstraelen, L. Submanifolds Theory-A Contemplation of Submanifolds, "Frontmatter" in Geometry of Submanifolds, AMS Contemporary Mathematics; volume in honor of Professor Bang-Yen Chen; Van der Veken, J., Cariazo, A., Suceava, B.D., Oh, Y.M., Vrancken, L., Eds.; American Mathematical Society: Providence, RI, USA, 2020.

10. Chen, B.Y. Geometry of Submanifolds; Marcel Dekker Publ. Co.: New York, NY, USA, 1973.

11. Chen, B.Y. Riemannian submanifolds. In Handbook of Differential Geometry; Dillen, F.J.E., Ed.; Elsevier: Amsterdam, The Netherlands, 2000; Volume 1, Chapter 3, pp. 187-418.

12. Rouxel, B. Sur quelques propriétés anallagmatiques de l'espace euclidien $\mathbb{E}^{4}$. In Mémmoire Couronné; Acad. Royale Belge: Brussels, Belgium, 1982; 128p.

13. Chen, B.Y.; Ogiue, K. On totally real submanifolds. Trans. Am. Math. Soc. 1974, 193, 257-266. [CrossRef]

(C) 2020 by the authors. Licensee MDPI, Basel, Switzerland. This article is an open access article distributed under the terms and conditions of the Creative Commons Attribution (CC BY) license (http:/ / creativecommons.org/licenses/by/4.0/). 